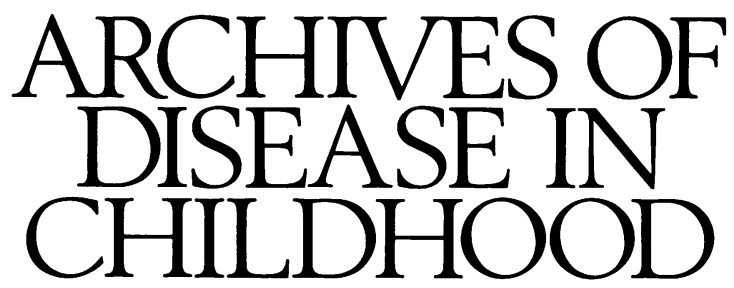

\title{
Cerebral ultrasound and neurological impairment: telling the future
}

After death, severe neurodevelopmental disability is the most feared complication of neonatal intensive care. Over the last 10 years, the practice of regularly scanning the brain of tiny babies has developed in the belief that this will allow the staff to monitor it realistically for signs of major complications. It is therefore implicit that knowledge of these abnormalities correlates with an accurate prediction of outcome. This annotation reviews our current understanding of the significance of cerebral ultrasound abnormalities detected in the neonatal period with reference to subsequent outcome.

Ultrasound imaging provides an acoustic picture of the infant's brain; the appearance being related to the reflective properties of various cerebral structures. The production of such images raises the possibility of systematic errors if there is only limited agreement on the diagnostic criteria for ultrasound abnormalities. Pathology as diverse as haemorrhage, gliosis, calcification, lipomata, and cerebral oedema may all produce echos of similar character, although their distribution may vary. The interpreter of the ultrasound image must base the diagnosis on good studies of pathological correlation. Even with accurate diagnosis, accurate prognosis depends on the frequency and timing of the scans together with the spatial resolution of the ultrasound machine. This in turn depends on the transducer frequency and the age of the equipment.

Before attempting to assess the reliability of ultrasound in predicting outcome, it is necessary to define the questions that the paediatrician wishes to ask of the technique. Major neurodevelopmental impairments such as cerebral palsy, mental retardation, and cortical blindness are often devastating and untreatable conditions that may be due in some cases to haemorrhagic or ischaemic insults during the early neonatal period. At present, with the exception of posthaemorrhagic hydrocephalus, there is no prospect of treating neonatal cerebral insults once they have occurred and therefore ultrasound diagnosis is used arguably as a screening test for selective withdrawal of care. Paediatricians will elect to continue intensive care in many infants and then the value of regular ultrasound is more towards reassurance or preparing the parents for the risk of subsequent major disability. Although ultrasound may predict some cases of major disability, particularly cerebral palsy, it is unreasonable to expect that when used early in the neonatal period, particularly in very immature infants, ultrasound will or should predict minor impairment which is only detectable after the age of 5 years or more. This is not to argue that attention deficit, clumsiness, or learning problems are not important disabilities, but without effective management or intervention therapies and accepting that withdrawal of care is not appropriate for these conditions, there is little clinical point at the present time to recognising an ultrasound appearance that may predict their development.

It is important to make a distinction between the appearances seen on ultrasound examination and the underlying pathology. Some authors report the correlation between ultrasound abnormalities (without implying the need to define the underlying condition) and outcome and others infer the pathological process (perhaps incorrectly) and correlate this with outcome. As far as possible I will attempt to relate ultrasound appearances, as distinct from implied diagnoses, to subsequent outcome.

\section{Normal scans}

There is remarkable consensus in the literature that consistently normal ultrasound scans in premature infants who have received intensive care accurately predicts normal outcome. Most studies cite a risk of major disability in the order of $5 \%$ (95\% confidence intervals of $1-10 \%)$ for premature infants who have been regularly scanned and who have showed no evidence of cerebral abnormality. ${ }^{2}$ Parents and staff can be strongly reassured that these babies are at very low risk for the subsequent development of cerebral palsy or severe developmental delay.

\section{Germinal matrix haemorrhage-intraventricular haemorrhage}

The ultrasound diagnosis of germinal matrix haemorrhageintraventricular haemorrhage (excluding involvement of the periventricular white matter) is now well accepted and most studies comparing ultrasound with pathological specimens report very good correlation. There is consistent agreement that echos apparently confined to the region of the germinal matrix alone carry no increased risk of adverse outcome. The ultrasound appearance referred to as 'uncomplicated periventricular haemorrhage' comprising echos in or around the lateral ventricle(s), ${ }^{2}$ but not associated with either parenchymal echodensities or subsequent ventricular dilatation, has been shown in two large cohort studies to have no increased risk of adverse outcome. ${ }^{12}$ Others suggest that the risk of subsequent disability is related to the extent that the ventricles are distended by the echos, ${ }^{3}{ }^{4}$ but this association may be due to the fact that late parenchymal lesions occur in up to $27 \%$ of those infants with the ultrasound appearance of intraventricular haemorrhage diagnosed in the first week of life. ${ }^{3}$ In summary, the outcome associated 
with the ultrasound appearance of isolated germinal matrix haemorrhage-intraventricular haemorrhage, without parenchymal involvement, is very good. At best the prognosis is excellent and at worse the diagnosis only slightly increases the risk of severe disability compared with an infant of the same degree of prematurity with consistently normal ultrasound appearances.

\section{Non-cavitating echodensity}

This condition has been described in the literature under a number of names including 'prolonged flare', 56 'small intraparenchymal echodensity', 7 'peritrigonal echogenic blush', 8 and 'echogenic periventricular halo', 9 but unfortunately there is no consistent diagnostic agreement. It is accepted that some degree of echodensity seen in either coronal or parasagittal plane is extremely common in premature infants and is often normal. Those features that make the ultrasound appearance more likely to be significant are persistence on repeated scans, consistent or coarse pattern, and the extent of the anatomical distribution of echodensity. Pathological correlation is difficult as babies with this condition rarely die and the few available correlative studies report reasonably good accuracy. Using fairly rigorous ultrasound diagnoses and comparing the scan findings with periventricular leucomalacia detected at necropsy the accuracy ranges between $70-81 \%{ }^{8} 10$ There is limited follow up data available for infants with non-cavitating persistent echodensity, ${ }^{167}$ and the studies are not directly comparable because of differences in the ultrasound definitions, but overall the prognosis appears to be relatively good. In two studies, only 8-9\% of infants with this appearance had cerebral palsy diagnosed at follow up of 18 months' duration. ${ }^{17}$ This is not significantly higher than the risk of cerebral palsy in any group of prematurely born infants with no ultrasound abnormalities. The third study reported cerebral palsy in $17 \%$ of children with 'flare' lasting 10 days or more and followed up to 24 months. ${ }^{6}$ It is possible that the ultrasound appearance of non-cavitating echodensity will be found to correlate with less severe forms of motor impairment than cerebral palsy, but these results are not yet available. This ultrasound appearance seems to have a good prognosis and it is unlikely to be associated with severe cerebral palsy.

\section{Periventricular cavitation}

Two forms of echolucent cavitation are recognised on ultrasound examination. In one the cavities (or cysts as they are commonly labelled) are small, often multiple, and separate from the lateral ventricles and the condition is referred to as cavitating periventricular leucomalacia. In the other the echolucent cavity is single, large, and in direct communication with the lateral ventricle; this is commonly diagnosed as porencephaly.

\section{Cavitating periventricular leucomalacia}

The ultrasound appearances of cavitation develop approximately 14 days or more after the first appearance of the echodense lesion associated with the acute pathological process and is therefore usually a late sign. In general terms, this ultrasound appearance carries a poor prognosis, but the accuracy of the prediction can be refined by attention to the size, number, and position of the echolucent cavities. Infants with evidence of cavities in the periventricular white matter, but who do not show signs of cerebral palsy (albeit at a relatively short follow up interval) have either a single ${ }^{1}$ or a number of small cavities ( $<3 \mathrm{~mm}$ in size). ${ }^{11} 12$ In addition, lesions confined to the frontal or parietal areas of the periventricular white matter may be associated with less severe forms of cerebral palsy. ${ }^{11}$ In contrast, lesions of the occipital periventricular white matter, ${ }^{1}$ and infants with bilateral cavitating lesions, ${ }^{3}$ have a very high risk of severe motor impairment.

Unfortunately, scans performed in the first week or two of life may not show cavitation, as the pathological process is in the process of evolving. Before the development of cysts, parenchymal echodensity is often evident, but the end stage ultrasound lesion may be in doubt. It has been shown that the positive predictive value for neurodevelopmental outcome at one year is $100 \%$ when white matter abnormalities are present at $\mathbf{4 0}$ weeks' postconceptual age, compared with $75 \%$ when the periventricular echodensity was present at two weeks after birth. ${ }^{13}$ In babies with extensive echodensity involving the periventricular white matter detected during the first week or two of life, the underlying cause may not be inferred. Two major forms of pathology exist: periventricular leucomalacia and venous infarction (see below).

\section{Porencephaly}

A characteristic late ultrasound finding is a single large echolucent cavity widely communicating with the ipsilateral lateral ventricle. It is almost always unilateral and is often referred to as porencephaly. Although the ultrasound appearance is usually unmistakable, its pathogenesis remains somewhat controversial. Opinion is strengthening towards it representing the end stage of periventricular venous infarction, as it is almost invariably preceded or associated with germinal matrix haemorrhage on the same side as the lesion. The major difficulty with the ultrasound diagnosis of venous infarction is that it must be inferred from an appearance which, in its early stages, may be similar to that of evolving non-cavitating echodensity or periventricular leucomalacia. This is the likely reason that early abnormal ultrasound appearances (those present in the first two weeks of life) are less good at predicting adverse outcome than those made later. ${ }^{2} 313$

The prognostic importance of porencephaly is controversial. Many authors lump porencephalic lesions together with cavitating periventricular leucomalacia and relate extensive periventricular abnormalities of both types to adverse outcome. ${ }^{2}$ Those that have considered porencephaly separately suggest that it is very likely to be associated with cerebral palsy ${ }^{314}$ as well as developmental impairment. ${ }^{14}$ Cooke suggests that cerebral palsy is more likely to follow porencephaly than cavitating periventricular leucomalacia. ${ }^{3}$ This is disputed by others who suggest that porencephaly is a much more benign condition, ${ }^{15}$ either unassociated with cerebral palsy in most or a hemiplegia associated with normal development in the rest. Further prospective follow up studies are necessary to resolve the prognostic importance of ultrasound detected porencephaly.

\section{Conclusion}

There is no doubt that consistently normal ultrasound scans, particularly if a late (40 weeks' postconceptual age) scan is available, are very reassuring for the baby's medical and nursing attendants and the parents can be informed of the strong likelihood that their baby will have no major neurodevelopmental sequelae. Multiple cavitating echolucent lesions in the periventricular white matter carry a poor prognosis, but the significance of a porencephalic cavity remains uncertain. There is no doubt that the prognostic accuracy of late ultrasound scans is far better than early scans performed in the first week of life and this limits the value of the technique as a reliable method for recognising 
the infant in whom selective withdrawal of intensive care is a realistic and honest option.

\author{
Department of Paediatrics \\ and Child Health, \\ University of Leeds, \\ Clarendon Wing, \\ The General Infirmary at Leeds, \\ Leeds LS2 9NS \\ 1 Graham M, Levene MI, Trounce JQ, Rutter N. Prediction of cerebral palsy \\ in very low birthweight infants: prospective ultrasound study. Lancet 1987 \\ ii: $593-6$. \\ 2 Stewart AL, Reynolds EOR, Hope PL, et al. Probability of neurodevelop- \\ mental disorders estimated from ultrasound appearance of brains of very \\ mental disorders estimated from ultrasound appearance
preterm infants. Dev Med Child Neurol 1987;29:3-11. \\ 3 Cooke RWI. Early and late cranial ultrasonographic appearances and outcome \\ in very low birthweight infants. Arch Dis Child 1987;62:931-7. \\ 4 Tudehope DI, Masel J, Mohay H, et al. Neonatal cranial ultrasonography as \\ predictor of 2 year outcome of very low birthweight infants. Aust Paediat \\ f 1989;25:66-71. \\ 5 Trounce JQ, Rutter N, Levene MI. Periventricular leucomalacia and intra- \\ ventricular haemorrhage in the preterm neonate. Arch Dis Child 1986;61: \\ 1196-202.
}

M I LEVENE

\section{Incidence and prevalence}

The terms incidence and prevalence are occasionally used as though they are synonymous, this, however, is to misuse them because, not only do they have quite distinct meanings but there is an arithmetical relationship between them.

The point prevalence of a given condition is the number of cases in a specific population at a point in time. The incidence is the number of new cases appearing in the population over a specified period of time. An analogy may be drawn with water flowing into and draining out of a basin. The quantity of water in the basin is equivalent to the prevalence and the water flowing in is the incidence. Obviously the level of water in the basin (the prevalence) will vary according to the rate at which water is flowing in (the incidence) and the rate it is flowing out (which can be expressed by the duration of the disease). Thus: prevalence $\propto$ incidence $\times$ duration. If two conditions are met, namely that: (a) the incidence rate is constant (for most disease conditions this is a reasonable assumption but in an epidemic situation-for example, the cyclical variation found with many communicable diseases-this condition will not be met) and (b) the mean duration of the disease is constant, then prevalence $=$ incidence $\times$ duration $(P=I \times D)$.

The relevance of this relationship to paediatric practice may be considered at both general and specific levels. At the general level I regularly ask medical undergraduates for examples of outstanding successes in the medical therapeutic armamentarium and how have these successes affected the prevalence of disease. It is salutary that some have no ready answer while disconcertingly few mention antibiotics or insulin. Almost invaribly they state that successful therapeutic measures reduce the prevalence of disease. While this may be true for some conditions-for example, tuberculosis where both incidence and duration of active disease have been dramatically curtailed after the introduction of antituberculous drugs-most of today's diseases have increased in prevalence as a result of successful treatment. Conditions that were previously incompatible with survival beyond childhood are now found in adults and even in second childhood. Juvenile diabetes, cystic fibrosis, end stage renal failure, severe mental and/or physical impairment all come to mind as examples. Indeed, medicine today may be considered the victim of its own success. The changing pattern of disease in the general population from acute to chronic with all the pressure this change exerts on National Health Service resources is, at
6 de-Vries LS, Regev R, Pennock JM, Wigglesworth JS, Dubowitz LM. Ultrasound evolution and later outcome of infants with periventricular densities. Early Hum Dev 1988;16:225-33.

7 McMenamin JB, Shackelford GD, Volpe JJ. Outcome of neonatal intraventricular hemorrhage with periventricular echodense lesions. Ann Neurol 1984; 15:285-90.

8 DiPietro MA, Brody BA, Teele RL. Peritrigonal echogenic 'blush' on cranial sonography: pathologic correlates. AJR 1986;146:1067-72.

9 Grant EG, Schellinger D, Richardson JD, Coffey ML, Smirniotopoulous JG. Echogenic periventricular halo: normal sonographic finding or neonatal cerebral hemorrhage. $A \mathcal{F} R$ 1983;140:793-6.

10 Trounce JQ, Fagan D, Levene MI. Intraventricular haemorrhage and periventricular leucomalacia: ultrasound and autopsy correlation. Arch Dis Child 1986;61:1203-7.

11 Fawer C-L, Calame A, Furrer M-T. Neurodevelopmental outcome at 12 months of age related to cerebral ultrasound appearances of high risk premonths of age related to cerebral ultrasound appen

12 Graziani LJ, Pasto M, Stanley C, et al. Neonatal neurosonographic correlates of cerebral palsy in preterm infants. Pediatrics 1986;78:88-95.

13 Nwaesei CG, Allen AC, Vincer MJ, et al. Effect of timing of cerebral ultrasonography on the prediction of later neurodevelopmental outcome in sonography on the prediction of later neurodevelopm
high-risk preterm infants. $\mathcal{F}$ Pediatr 1988;112:970-5.

14 Guzzetta F, Shackelford GD, Volpe S, Perlman JM, Volpe JJ. Periventricular intraparenchymal echodensities in the premature newborn: critical determinant of neurologic outcome. Pediatrics 1986;78:995-1006.

15 De-Vries LS, Dubowitz LMS, Dubowitz V, et al. Predictive value of cranial ultrasound in the newborn baby: a reappraisal. Lancet 1985;ii:137-40.

least in part, due to an increase in prevalence of many diseases, the consequences of effective medical intervention.

At a more specific level, the relationship between incidence and prevalence must be invoked in the debate on the levels of impairment and disability associated with improved survival of low birthweight infants-that is, in the outcome audit of obstetric and neonatal intensive care.

First, it is not valid to refer to incidence for any disease in which the aetiological agent is thought to act prenatally unless it is possible to determine how many affected conceptuses were aborted or lost as stillbirths. At the best, all that can be determined for these conditions is the prevalence at birth. The congenital anomalies and probably the vast majority of cases of cerebral palsy fall into this category. Currently the debate centres around reports that there is an increasing prevalence of cerebral palsy among very low birthweight infants and how the increase is related to either obstetric and/or neonatal management. If the prevalence is increasing it can be due to an increase in either incidence or duration, or both.

To take duration first, the assumption has to be that the infant is brain damaged prepartum and changes in obstetric/ neonatal management merely improve survival of affected infants-that is, prevalence is increased because of a greater duration of the condition. This being so, further advances in care can be expected to result in a higher prevalence of cerebral palsy.

The alternative explanation is that prevalence is increasing because of an increased incidence-that is, new cases are being produced as a result of brain damage inflicted in the peripartum period. Implicit in this explanation is that peripartum management must, in some way, have been suboptimal with all the medicolegal spectres this raises.

Recently there has been discussion about whether demonstrable intracranial lesions, for example, periventricular leucomalacia or intraventricular haemorrhage associated with anoxic or other insults cause the cerebral palsy, or whether these insults are more prone to occur in the infant whose brain has already been compromised. In this context, Koch's postulates need to be applied to cerebral palsy.

Department of Public Health,

P O D PHAROAH

University of Liverpool,

PO Box 147

Liverpool L69 3BX 